\section{Engineering Science of Polymeric Materials}

\section{Z.H. Stachurski \\ (Polymer Division of the Royal Australian \\ Chemical Institute, 1987)}

This book contains a practical approach to "polymers as received" for engineering purposes, particularly for the engineer or scientist who needs a materials approach to polymers, but does not intend to make a career in the field. In addition, it is a valuable introductory reference book, able to serve as a starting point for common problems in developing polymer materials. To use and understand the information and methodology in Eng. Sci..., one would need a few supplemental books, such as Flory's Principles of Polymer Chemistry, Tadmor and Gogos' Principles of Polymer Processing, and the Polymer Handbook. The level of the book is eminently suitable for teaching junior, senior or first-year graduate students. I know of no other polymer book which could be so readily used for engineering.

The book is divided into four main sections. The first is an introduction to polymers, methods of classification, good descriptions of characterization methods, phase diagrams, and detailed descriptions of specific uses. The second discusses processes involved in phase transitions of polymers on the macro and molecular level. The third covers mechanical behavior and the fourth methods to improve mechanical properties.

In all cases, Stachurski has covered the items with the minimum set necessary for completeness. For example, in the section on characterization methods, a general engineering approach designed to elucidate the properties of a polymeric material is outlined in order: first, calorimetry (in this case, DSC) and dilatometry to determine macroscopic bulk properties; then, infrared and nuclear magnetic resonance spectroscopies to determine the internal chemical character; gel permeation chromatography (GPC) for determining the polymer molecular weight distribution; and finally, microscopy and $x$-ray diffractometry to determine the final structure of the solid. The principle and methods for each technique are discussed briefly, with references given for more detail. He devotes more space to DSC and GPC than to the other techniques, but probably because these are not generally described in analytical chemistry textbooks.

An area I felt was not sufficiently covered was properties of solutions of polymers, which may become important during the crosslinking of a dilute gel or in the process of isolating more narrow molecular weight distributions of the polymer chains. One more negative comment is that the production quality of the book is lacking. A few pages were out of order and some of the print is light. Overall, however, Stachurski has given us a good introductory book to his title, "Engineering Science of Polymeric Materials."

Lucy $M$. Hair is a staff scientist, Chemistry $\mathcal{E}$ Materials Science Department, Lawrence Livermore National Laboratory. She has developed organic foams for the Laser Fusion Energy Program, performed independent research on the crosslinking of rigid-rod polymers and is currently working on catalyst development for methane conversion to liquids.

\section{Hydrogen in Intermetallic Compounds I}

\section{Edited by L. Schlapbach \\ (Springer-Verlag, 1988)}

Hydrogen in Intermetallic Compounds I provides useful, easily read reviews of techniques for preparing and hydriding intermetallics and for characterizing the resulting compounds. The demonstrated capability of intermetallics to store and release large quantities of hydrogen has catalyzed interdisciplinary studies in this field.

The complexity and variety of ternary metal hydrides available for study are summarized in Chapter 4 . The chapter concludes that our understanding of the hydriding tendencies of such compounds is developed to the point that hydrogen

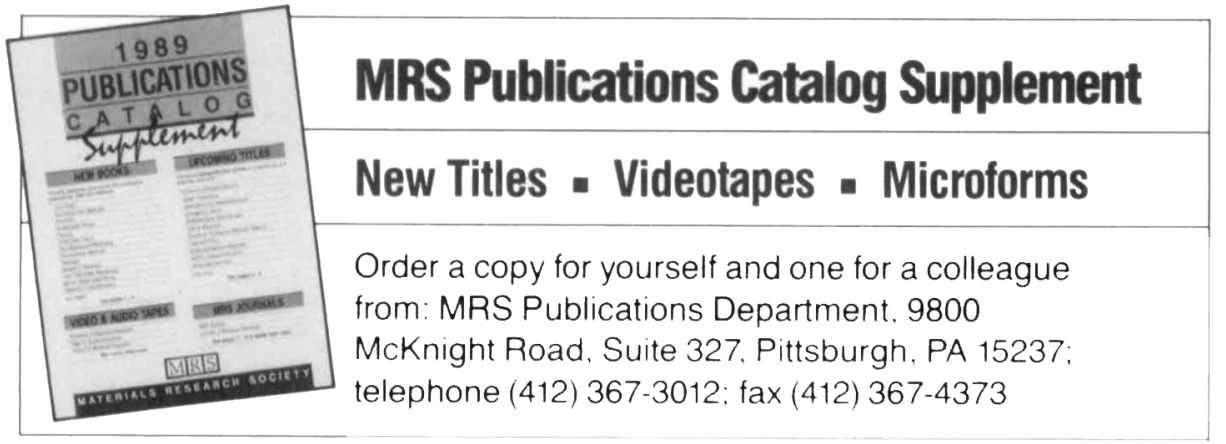

"occupancies can be rationalized in terms of geometrical factors, attractive nearestneighbor interactions and electronic factors thus providing a qualitative understanding ..." The words rationalized and qualitative were well chosen and appropriate. Such words were also applied to the characterization of electronic properties in Chapter 5, which states that "good qualitative, and in many examples even quantitative, agreement between band-structure calculations for metal hydrides and measured spectra" was developed.

Chapter 6 discusses the understanding of the heats of formation of the hydrides and concludes that "to rationalize trends in hydride stability and site occupation, several empirical correlations have been proposed..." Chapter 7 describes magnetic properties, Mössbauer effect and superconductivity and states that "magnetism in the $3 \mathrm{~d}$ elements and their intermetallics remains a controversial topic and is by no means a solved problem."

It was refreshing to read scholarly reviews that present a wealth of data, provide an excellent source of references and challenge the reader by calling speculation, "speculation," and empiricism, "empiricism."

The chapter on the thermodynamics of intermetallic compound-hydrogen systems concludes that "considerable improvements in both experimental and theoretical investigations can be anticipated in the next few years." Such improvements are anticipated because the foundations developed in the large volume of literature reviewed provide a solid base for future studies. Chapter 2 describes the preparation of intermetallics and hydrides and states that "we hope we have illustrated the importance of the metallurgical state of the base intermetallic compounds which will be used for research and application purposes." All seven of the reviews in this volume illustrate the importance of, and potential for, intermetallic compounds and their hydrides.

This book will be a valuable reference for workers in hydrogen energy, fusion, electrochemical cell technology, catalysis, getters, and perhaps even superconductivity. Each chapter clearly illustrates that the field of hydrogen in intermetallic compounds is wide open for developments in experiment, analysis and understanding. The book provides a useful guide to aid in such development.

M.R. Louthan, Jr. is a senior research associate, Materials Technology Division, Westinghouse Savannah River Company. He has studied hydrogen-metal systems for approximately 25 years and has over 100 publications in this field. 